\title{
Controlled-release of tetracycline and lovastatin by poly(D,L-lactide-co-glycolide acid)-chitosan nanoparticles enhances periodontal regeneration in dogs
}

\author{
This article was published in the following Dove Press journal: \\ International Journal of Nanomedicine \\ 18 January 2016 \\ Number of times this article has been viewed
}

\author{
Bor-Shiunn Lee' \\ Chien-Chen Lee ${ }^{2}$ \\ Yi-Ping Wang ${ }^{2}$ \\ Hsiao-Jan Chen ${ }^{3}$ \\ Chern-Hsiung Lai ${ }^{4}$ \\ Wan-Ling Hsieh' \\ Yi-Wen Chen ${ }^{2}$ \\ 'Graduate Institute of Oral Biology, \\ ${ }^{2}$ Graduate Institute of Clinical \\ Dentistry, School of Dentistry, \\ National Taiwan University and \\ National Taiwan University Hospital, \\ ${ }^{3}$ Department of Clinical Laboratory \\ Sciences and Medical Biotechnology, \\ College of Medicine, National Taiwan \\ University, Taipei, ${ }^{4}$ College of Life \\ Science, Kaohsiung Medical University, \\ Kaohsiung, Taiwan
}

Correspondence: Yi-Wen Chen Graduate Institute of Clinical Dentistry, School of Dentistry, National Taiwan University and National Taiwan University Hospital, No I, Chang-Te St, Taipei 10048, Taiwan

$\mathrm{Tel}+886223123456$

Fax +886 $22383 \quad$ I346

Email yiwenchen@ntu.edu.tw
Abstract: Chronic periodontitis is characterized by inflammation of periodontal tissues, leading to bone resorption and tooth loss. The goal of treatment is to regenerate periodontal tissues including bone and cementum lost as a consequence of disease. The local delivery of tetracycline was proven to be effective in controlling localized periodontal infection without apparent side effects. Previous studies suggested that lovastatin has a significant role in new bone formation; however, the local delivery of lovastatin might enhance its therapeutic effects. A number of local delivery devices have been developed recently, including poly(D,L-lactide-co-glycolide acid) (PLGA) nanoparticles. The aim of this study was to develop a local delivery device, PLGA-lovastatin-chitosan-tetracycline nanoparticles, which allows the sequential release of tetracycline and lovastatin to effectively control local infection and promote bone regeneration in periodontitis. The size and microstructure of nanoparticles were examined by transmission electron microscopy, Nanoparticle Size Analyzer, and Fourier transform infrared spectroscopy. The release of tetracycline and lovastatin was quantified using a UV-Vis spectrophotometer. Furthermore, the cytotoxic effect and alkaline phosphatase activity of the nanoparticles in osteoblast cell cultures as well as antibacterial activity against periodontal pathogens were investigated. Finally, the bone regeneration potential of PLGA nanoparticles in three-walled defects in beagle dogs was investigated. The results indicated that PLGA-lovastatin-chitosantetracycline nanoparticles showed good biocompatibility, antibacterial activity, and increased alkaline phosphatase activity. The volumetric analysis from micro-CT revealed significantly increased new bone formation in defects filled with nanoparticles in dogs. This novel local delivery device might be useful as an adjunctive treatment in periodontal regenerative therapy.

Keywords: lovastatin, tetracycline, chitosan, poly(D,L-lactide-co-glycolide acid), periodontal regeneration

\section{Introduction}

Periodontitis is a chronic infectious disease resulting in inflammation of the supporting tissues, progressive attachment loss, and bone resorption. It is highly prevalent and can affect up to $90 \%$ of the worldwide population and is considered the major cause of tooth loss in adults. ${ }^{1,2}$ Surgical techniques, ${ }^{3-5}$ mechanical debridement of the denuded root surface, ${ }^{3,6}$ and local or systemic application of antimicrobial agents ${ }^{7-11}$ are currently used treatments for periodontitis. The use of systemic antibiotics in the treatment of moderate to severe periodontitis has been discussed previously. ${ }^{7,8}$ However, the repeated and long-term use of systemic drugs has potential side effects and may lead to the development of bacterial resistance and inadequate antibiotic concentrations in the 
periodontal tissue and gingival crevicular fluid. ${ }^{7,8}$ Complete eradication of the pathogenic organisms from infected sites has not been achieved by surgical and mechanical treatments. Therefore, the local delivery of antibiotics into the periodontal pocket is becoming more prevalent to overcome these limitations and ensure an effective concentration of antibiotics at infection sites. ${ }^{9-11}$

Local drug delivery systems have been used as an adjunct to conventional scaling and root planing, particularly in sites with deep periodontal pockets. ${ }^{9-11}$ However, there are only a limited number of products commercially available. Ideal local drug delivery systems deliver drugs into periodontal pockets, as well as maintaining an effective drug concentration at target sites. Chitosan, a natural cationic polysaccharide, is a nontoxic, biocompatible, and biodegradable polymer with multiple pharmaceutical applications including drug delivery, cosmetics, and tissue engineering. ${ }^{12,13}$ In a previous in vivo study, chitosan particles inhibited the growth of both Aggregatibacter actinomycetemcomitans (A. actinomycetemcomitans) and Porphyromonas gingivalis and modulated inflammatory responses in human gingival fibroblasts. ${ }^{14}$ Furthermore, chitosan film enriched with an antioxidant agent, taurine, stimulated macrophage and fibroblast activity, further accelerating wound healing in fenestration defects in beagle dogs. ${ }^{15}$ Metronidazole-loaded chitosan microparticles were successfully prepared by an emulsion cross-linking method and might be suitable for future periodontitis treatment. ${ }^{16}$ A clinical study reported that chitosan gel incorporated with or without $15 \%$ metronidazole was effective in probing depth reduction in patients with chronic periodontitis. ${ }^{17}$ Taken together, these studies indicate that chitosan is potentially biocompatible and might be a safe and effective delivery system.

Statins are lipid-lowering drugs that have become a mainstay in the treatment of high cholesterol to prevent cardiovascular and cerebrovascular events related to increased lipid levels. ${ }^{18,19}$ Statins have other effects including antiinflammatory and antioxidant properties, the stimulation of bone formation by increasing the expression of the BMP-2 gene in bone cells, and inducing angiogenesis thus providing a new direction in the field of periodontal therapy..$^{20,21}$ The application of statins was associated with decreased tooth loss in chronic periodontitis patients. ${ }^{22}$ In a rat model, the topical application of statins prevented periodontal tissue breakdown and had beneficial effects on alveolar bone recovery after ligature-induced alveolar bone resorption. ${ }^{23}$ Poly(D,Llactide-co-glycolide acid) (PLGA) is a hydrophobic synthetic polymer with good biodegradability and biocompatibility.
PLGA is the most popular available biodegradable polymer because of its favorable degradation characteristics, possibilities for sustained drug delivery, and long clinical experience..$^{24,25}$ The encapsulation of simvastatin in PLGA microspheres loaded into hydrogel achieved a favorable drug-release profile and enhanced bone remodeling in vitro. ${ }^{26}$ Further in vivo experiments revealed that simvastatin loaded PLGA microspheres significantly enhanced bone formation in rabbit calvaria critical size defects, ${ }^{27}$ and that lovastatin released from PLGA nanoparticles enhanced bone repair in rats. ${ }^{28}$ In randomized clinical trials, locally administered simvastatin significantly improved the clinical outcomes of scaling and root planing for treating mandibular buccal Class II defects, and in patients with chronic periodontitis, even when they were smokers or with type II diabetes. ${ }^{29-31}$

The aim of this study was to prepare novel PLGAlovastatin-chitosan-tetracycline nanoparticles for the local delivery and controlled release of tetracycline and lovastatin. The cytotoxicity, alkaline phosphatase (ALP) activity, and antibacterial activity were evaluated. In addition, the bone regeneration potential of fabricated nanoparticles was examined using three-walled defects in beagle dogs.

\section{Materials and methods Materials}

PLGA(L/Gmolarratio of 50:50, molecularweight $=30-60 \mathrm{kDa})$, chitosan ( $85 \%$ deacetylated, molecular weight $=190-310 \mathrm{kDa})$, tetracycline hydrochloride, poly(vinyl alcohol) (molecular weight $=89,000-98,000 \mathrm{kDa}$ ), chloroform, acetic acid, and acetonitrile were purchased from Sigma-Aldrich Co. (St Louis, MO, USA).

\section{Preparation of PLGA-lovastatin-chitosan- tetracycline nanoparticles}

PLGA-lovastatin-chitosan-tetracycline nanoparticles were prepared by modification of methods reported previously. ${ }^{28}$ Briefly, $0.12 \mathrm{~g}$ PLGA and $4 \mathrm{mg}$ lovastatin were dissolved in chloroform to form a $3 \mathrm{wt} \%$ PLGA solution. Chitosan was dissolved in $0.5 \mathrm{M}$ acetic acid and the mixture was then added to the poly(vinyl alcohol) solution containing tetracycline. A solution ( $\mathrm{W}_{2}$ phase) containing $0.3 \mathrm{wt} \%$ chitosan, $1 \mathrm{wt} \%$ poly(vinyl alcohol), and three concentrations $(0.1,0.3$, $0.5 \mathrm{wt} \%$ ) of tetracycline was prepared. Phosphate-buffered saline (PBS) $(800 \mu \mathrm{L})$ was added to $4 \mathrm{~mL}$ of PLGA and the resultant solution was emulsified at $3.5 \mathrm{~Hz}$ for 2 minutes using a probe sonicator (Misonix Sonicator 3000, Misonix, Inc., Farmingdale, NY, USA). This $\mathrm{W}_{1} / \mathrm{O}$ emulsion was then poured into $24 \mathrm{~mL}$ of $\mathrm{W}_{2}$ phase and emulsified at $6.5 \mathrm{~Hz}$ 
for 10 minutes. After the evaporation of chloroform, the polymer was precipitated, the nanoparticles were isolated by centrifugation, and then washed with deionized water several times.

\section{Morphological examination and particle size measurement}

The morphology of PLGA, PLGA-chitosan, and PLGAlovastatin-chitosan-tetracycline nanoparticles was examined by transmission electron microscopy (JEM-1400, JEOL Ltd., Tokyo, Japan) at an accelerating voltage of $80 \mathrm{kV}$. Nanoparticles treated with $1 \%$ uranyl acetate on copper grids were used for negative staining. Particle size was measured via dynamic light scattering analyses using a 90Plus Nanoparticle Size Analyzer (Brookhaven Instruments Corporation, Holtsville, NY, USA). The particle size measurements were conducted at a fixed $90^{\circ}$ angle, a wavelength of $632.8 \mathrm{~nm}$, and temperature of $25^{\circ} \mathrm{C}$. For all measurements, specimens were equilibrated in a temperature-controlled chamber for 5 minutes and then dynamic light scattering measurements were promptly started. Data collection and analysis were performed using BIC software (Brookhaven Instruments Corporation) with a dust filter cutoff value setting at 30 .

\section{Fourier transform infrared spectroscopy}

PLGA, chitosan, and PLGA-chitosan nanoparticles were characterized using Fourier transform infrared spectroscopy (FTIR-4200, Jasco International Co., Ltd., Tokyo, Japan). Two mg of each sample was mixed with $200 \mathrm{mg}$ potassium bromide and the mixture was compressed into a $\mathrm{KBr}$ disc. Each $\mathrm{KBr}$ disc was scanned over a wavenumber region of $650-4,000 \mathrm{~cm}^{-1}$ with resolution of $4 \mathrm{~cm}^{-1}$.

\section{UV-Vis assay for the in vitro controlled release of lovastatin and tetracycline}

A UV-Vis spectrophotometer (Agilent 8453, Agilent Technologies, Santa Clara, CA, USA) was used to observe specific peaks used to identify tetracycline and lovastatin from the PLGA-chitosan, lovastatin, and tetracycline preparations. Wavelengths of 362 and $248 \mathrm{~nm}$ were chosen for the quantification of tetracycline and lovastatin, respectively, because they were not affected by acetonitrile and PLGA-chitosan. Tetracycline was dissolved in PBS and lovastatin was dissolved in acetonitrile and PBS with a volume ratio of $<0.01$. There was no precipitate of lovastatin and tetracycline in the standard solution as determined by light microscopy. To prepare a calibration curve, the concentration of tetracycline in a standard solution ranged from 0 to $40 \mathrm{mg} / \mathrm{mL}$ and the concentration of lovastatin ranged from 0 to $30 \mathrm{mg} / \mathrm{mL}$. The encapsulation efficiency of lovastatin and tetracycline was calculated using the following formula:

$$
\begin{gathered}
\text { Lovastatin encapsulation } \\
\text { efficiency }(\%)
\end{gathered}=\frac{\begin{array}{c}
\text { Initial (lovastatin) }- \\
\text { supernatant }(\text { lovastatin })
\end{array}}{\text { Initial }(\text { lovastatin })} \times 100 \%
$$

and

$$
\begin{aligned}
& \text { Initial (tetracycline) - } \\
& \begin{array}{c}
\text { Tetracycline encapsulation } \\
\text { efficiency }(\%)
\end{array}=\frac{\text { supernatant }(\text { tetracycline })}{\text { Initial }(\text { tetracycline })} \times 100 \%
\end{aligned}
$$

To measure the controlled release in vitro, $50 \mathrm{mg}$ of PLGA-lovastatin-chitosan-tetracycline nanoparticles was placed in a $50 \mathrm{~mL}$ PBS solution containing $0.1 \mathrm{wt} \%$ sodium azide and stirred at $100 \times g$ at $37^{\circ} \mathrm{C}$. At every time point, $0.5 \mathrm{~mL}$ solution was removed for measurement by UV-Vis reading at 362 and $248 \mathrm{~nm}$ for tetracycline and lovastatin, respectively.

\section{Cell culture and MTT assay}

Primary cultures of human bone marrow-derived osteoblasts (HOB) were established as described previously. ${ }^{28}$ Briefly, alveolar bone explants were harvested after obtaining informed consent from five healthy patients (aged 20-40 years) receiving odontectomy of the third molar. Permission to collect alveolar bone explants was obtained from the Ethical Committee of National Taiwan University Hospital (Case No 201105080RC). HOB cells were cultured in $\alpha$ MEM with $10 \%$ fetal bovine serum and $100 \mathrm{U} / \mathrm{mL}$ antibiotics (penicillin-streptomycin-amphotericin; SigmaAldrich Co.) at $37^{\circ} \mathrm{C}$ in $5 \% \mathrm{CO}_{2}$. The passage number was $8-12$. The cytotoxicity of PLGA-chitosan and PLGA-lovastatin-chitosan-tetracycline $(0.1,0.3,0.5 \mathrm{wt} \%)$ nanoparticles $(500 \mu \mathrm{g} / \mathrm{mL})$ was estimated in vitro using an 3-(4,5-dimethylthiazol-2-yl)-2,5-diphenyltetrazolium bromide (MTT) assay. HOB cells were seeded at a density of 3,000 cells/well and then incubated with nanoparticles at $37^{\circ} \mathrm{C}$. After 1-, 3-, and 5-day incubations, $20 \mu \mathrm{L}$ of MTT solution was added to each well and incubated for 3 hours. Subsequently, the medium was aspirated and $200 \mu \mathrm{L}$ of dimethyl sulfoxide was added to dissolve the formazan crystals. The optical density $\left(\mathrm{OD}_{570}\right)$ of the formazan solution was measured by microplate reader (ELx 800, BioTek, Winooski, VT, USA). 


\section{ALP activity}

HOB cells were seeded at a density of $2 \times 10^{4}$ cells per well and then incubated with nanoparticles at $37^{\circ} \mathrm{C}$. The ALP activity of PLGA-chitosan and PLGA-lovastatin-chitosan-tetracycline $(0.1,0.3,0.5 \mathrm{wt} \%)$ nanoparticles $(500 \mu \mathrm{g} / \mathrm{mL})$ was measured after 1-, 3-, 5-, 7-, and 9-day incubations by quantifying the conversion of $p$-nitro-phenylphosphate to $p$-nitrophenol. The fluorescence intensity was measured at an excitation wavelength of $356 \mathrm{~nm}$ and emission wavelength of $458 \mathrm{~nm}$. ALP activity was normalized to the total protein content of cells determined spectrophotometrically using a Micro BCA protein assay kit (Thermo Scientific, Waltham, MA, USA).

\section{Minimum inhibitory concentration test}

Minimum inhibitory concentrations for a panel of antibiotics were determined by the agar dilution method. Target bacteria were A. actinomycetemcomitans ATCC ${ }^{\circledR} 43719$ and Prevotella nigrescens (P. nigrescens) ATCC $^{\circledR}$ 33563. Bacteroides fragilis ATCC $^{\circledR} 25285$ and Bacteroides thetaiotaomicron ATCC $^{\circledR}$ 29741 were used as controls. Then, $4.3 \mathrm{~g}$ of Brucella agar (Difco Laboratories, Detroit, MI, USA) was added to $100 \mathrm{~mL}$ of distilled water and $0.1 \mathrm{~mL}$ of hemin and vitamin $\mathrm{K} 1$ solution to form a liquid medium. This was sterilized at $120^{\circ} \mathrm{C}$ in an autoclave (TOMIN, TM-326, Taipei, Taiwan) for 30 minutes and then sheep blood (BioMedia Laboratories, Petaling Jaya, Selangor Darul Ehsan, Malaysia) was added to form Brucella blood agar. Then, $13.5 \mathrm{~mL}$ of Brucella blood agar with hemin and vitamin $\mathrm{K} 1$ containing each concentration of antibiotic solution $(1.5 \mathrm{~mL})$ were added to each plate. Agar plates with different tetracycline concentrations $(0,0.031,0.0625,0.125$, $0.25,0.5,1,2,4,8,16,32,64$, and $128 \mathrm{mg} / \mathrm{mL}$ ) were submitted to antibiotic susceptibility tests. A standardized $0.5 \mathrm{McF}$ arland of the test strain culture was inoculated in a drop of solution across the surface of Brucella blood agar using a sterile cotton swab. The suspension matched or exceeded the density of the $0.5 \mathrm{McF}$ arland standard. A multipoint inoculator was used to deliver the suspension onto the surface. After 2-3 days incubation in an anaerobic environment, the minimum inhibitory concentration was defined as the lowest concentration of antibiotic at which there was no visible growth of the organism. The studies were repeated at least once on a separate day.

\section{In vitro antibacterial activity assay}

A. actinomycetemcomitans and $P$. nigrescens were cultured on anaerobic blood agar plates as previously described, and the inhibition zone was assessed. The antibacterial ability of four kinds of drugs, including PLGA-chitosan, PLGA-chitosan-lovastatin, PLGA-lovastatin-chitosantetracycline $0.1 \%$, and PLGA-lovastatin-chitosan-tetracycline
$0.3 \%$, was estimated by measuring the size of the inhibition zone. A $20 \mu \mathrm{L}$ volume of sample solution was prepared by adding the drugs to PBS ( $1 \mathrm{mg} / \mathrm{mL})$ after $0,1,3,5$, and 7 days, and was then dropped onto a $6 \mathrm{~mm}$ of blank disc (Blank Disc 50 disks, Creative Microbiologicals, Ltd., Taipei, Taiwan). The sample disc was then dried at room temperature for 10-20 minutes. A standardized 0.5 McFarland of the test strain culture was inoculated homogeneously on the surface of Brucella blood agar using a sterile cotton swab. Then, the sample discs were pasted onto the agar plate. The bacteria were incubated at $37^{\circ} \mathrm{C}$ for 48 hours and the inhibition zone for each sample on the plate was measured.

\section{Administration of PLGA-lovastatin- chitosan-tetracycline $0.3 \%$ nanoparticles to dogs}

Six healthy male 1.5-year-old beagle dogs, weighing $\sim 10 \mathrm{~kg}$, were used in this study. The protocol and procedures were approved by the Animal Research Center of National Taiwan University (No 20120500). All animal-handling procedures were performed according to the Guide for the Care and Use of Laboratory Animals of the National Institutes of Health and followed the guidelines of the Animal Welfare Act. All surgical procedures were performed under general and local anesthesia in sterile conditions. One clinician performed all surgery, including creating defects, placing nanoparticles, and wound closure. General anesthesia was achieved using a subcutaneous injection of $15 \mathrm{~mL}$ Zoletil $^{\circledR} 50$ (Virbac, Carros, France), $0.2 \mathrm{~mL}$ Rompun ${ }^{\circledR}$ (Bayer AG, Leverkusen, Germany), and $0.5 \mathrm{~mL}$ atropine. Local anesthesia was performed with $2 \%$ lidocaine hydrochloride $1.8 \mathrm{~mL}$ per cartilage containing epinephrine at a dilution of 1:10,000. All second and third incisors in the maxilla were extracted to assure adequate space for three-walled defects, and antibiotics (penicillin G, 200,000 U) were administered intramuscularly. After 8 weeks of healing, the mucoperiosteal flap was elevated, and threewalled intrabony defects $(4 \times 4 \times 5 \mathrm{~mm}$ : buccolingual, mesiodistal, and depth, respectively) were created on the mesial side of maxillary bilateral canines using round and fissure burs with a sterile saline coolant. The residual cementum was completely removed by scaling and root planing using Gracey curets. PLGA-lovastatin-chitosan-tetracycline $0.3 \%$ nanoparticles were prepared as a hydrogel by mixing with gelatin $\left(10 \mathrm{mg} / 100 \mathrm{~mm}^{3}\right)$, and were used to fill the defects by needle injection. The treatment group was randomly assigned and the defects were left unfilled in the control group. The mucoperiosteal flap was sutured by resorbable sutures. All wounds were kept clean by the topical application of $2 \%$ chlorhexidine gluconate three times a week for 8 weeks. 
Eight weeks after surgery, the animals were sacrificed. The blocks with defects were prepared and fixed in $10 \%$ buffered formalin and rinsed in PBS. After decalcification in Plank-Rychlo solution for 1 week, the blocks were trimmed, dehydrated, and embedded in paraffin. Serial sections of $5 \mu \mathrm{m}$ thickness were prepared in the mesiodistal plane, and then stained with hematoxylin and eosin at intervals of $100 \mathrm{~mm}$ and examined under a light microscope. A single examiner, masked to the group assigned, performed the following histomorphometric analysis.

\section{Reconstruction of bony defects using micro-CT}

Before fixation, tissue and bone blocks containing canine defects were trimmed to the proper size for the analysis chamber of a micro-CT device (in vivo micro-CT SkyScan1176, Bruker, Kontich, Belgium), and then the blocks were mounted on a turntable that could be shifted automatically in the axial direction. Six hundred projections were taken over 180 of object rotation $\left(0.3^{\circ}\right)$. The $\mathrm{X}$-ray shadow projections were digitized as 1,024×1,024 pixels with 256 gradations (8-bit). The spatial resolution obtained was $35.52 \mu \mathrm{m} / \mathrm{pixel}$. The transverse plane of images that were perpendicular to the tooth axis was chosen. A selected area of $120 \times 150$ pixel $=22.71\left(\mathrm{~mm}^{2}\right)$, similar to the defect size, was defined and calculated from the bottom of the defect (notch) toward the coronal area for 120 pixels ( $\sim 4 \mathrm{~mm})$. By summarizing of the net volume of the regenerated alveolar bone from the integrated volume of the selected areas of the periodontal defect (ImageJ), the newly formed bone volume (BV) and the total defect volume (TV) were obtained, respectively. The percentage of bone fill was determined as BV/TV (\%). CT examiner was masked to treatment rendered.

\section{Statistical analysis}

Data were expressed as mean $\pm \mathrm{SD}$, and mean values were used for statistical analyses using one-way analysis of variance. Post hoc comparisons were made using the Tukey-Kramer multiple comparison tests when the $P$-value was $<0.05$.

\section{Results}

\section{Particle size and infrared spectra of PLGA-lovastatin-chitosan-tetracycline nanoparticles}

Figure 1 shows the transmission electron microscopy photographs of PLGA, PLGA-chitosan, PLGA-lovastatin-chitosantetracycline nanoparticles, and the schematic diagram of PLGA-lovastatin-chitosan-tetracycline nanoparticles. The mean diameter of PLGA nanoparticles was $105.5 \mathrm{~nm}$.
The chitosan-coated PLGA nanoparticles had an increased mean diameter of $107.8 \mathrm{~nm}$. The morphology of PLGAlovastatin-chitosan-tetracycline nanoparticles was similar to that of PLGA-chitosan and the mean diameter was further increased to $111.5 \mathrm{~nm}$.

Figure 2 shows the infrared (IR) spectra of (Figure 2A) PLGA, (Figure 2B) chitosan, and (Figure 2C) PLGA-chitosan nanoparticles. The peaks at 1,751 and $1,082 \mathrm{~cm}^{-1}$ were identified as stretching vibrations of the $\mathrm{C}=\mathrm{O}$ and $\mathrm{C}-\mathrm{O}-\mathrm{C}$ groups in PLGA. ${ }^{32}$ The $3,336 \mathrm{~cm}^{-1}$ peak is the characteristic peak of $\mathrm{N}-\mathrm{H}$ and $\mathrm{O}-\mathrm{H}$ stretching vibrations in chitosan. In addition, the peaks at 1,649 and $1,560 \mathrm{~cm}^{-1}$ were identified as deformation vibrations of primary amine and secondary amine in chitosan. ${ }^{33}$ These characteristic peaks were also observed in the IR spectra of PLGA-chitosan.

\section{Drug release of PLGA-lovastatin-chitosan- tetracycline nanoparticles}

The calibration curves of lovastatin at $248 \mathrm{~nm}$ and tetracycline at $362 \mathrm{~nm}$ were done with $R^{2}=0.99757$ and 0.99968 , respectively. The lovastatin encapsulation efficiency (\%) of PLGA-lovastatin-chitosan-tetracycline $0.1 \%, 0.3 \%$, and $0.5 \%$ was $69.1 \pm 4.0,69.8 \pm 4.6$, and $70.3 \pm 4.3$, respectively. The tetracycline encapsulation efficiency was $10.6 \pm 2.7,22.4 \pm 3.1$, and 32.1 \pm 2.6 , respectively. A burst release of tetracycline was observed at the first day followed by gradual release until day 14 (Figure 3). An increased amount of tetracycline was released when the PLGA nanoparticles contained higher starting tetracycline concentration. Lovastatin release was slower than tetracycline and more prominent until day 21 .

\section{Cell toxicity and osteogenic potential of PLGA-lovastatin-chitosan-tetracycline nanoparticles}

The MTT assay showed no significant difference in cell viability between PLGA-chitosan and PLGA-lovastatinchitosan-tetracycline $0.1 \%, 0.3 \%$, and $0.5 \%$ for 1,3 , and 5 days of culture (Figure 4). Cells cultured with PLGAlovastatin-chitosan-tetracycline $0.1 \%$ or $0.3 \%$ nanoparticles expressed a significantly higher amount of ALP than other PLGA nanoparticles on days 7 and 9 (Figure 5). No significant difference was found between control and PLGAlovastatin-chitosan-tetracycline $0.5 \%$ groups.

\section{Bactericidal activity of PLGA-lovastatin- chitosan-tetracycline nanoparticles}

Figure 6 shows the inhibition zone of A. actinomycetemcomitans and P. nigrescens growth using PLGAlovastatin-chitosan-tetracycline $0.1 \%$ (Figure 6A) and 


\section{Particle size determined by dynamic light scattering}

Mean diameter $(\mathrm{nm})$

A

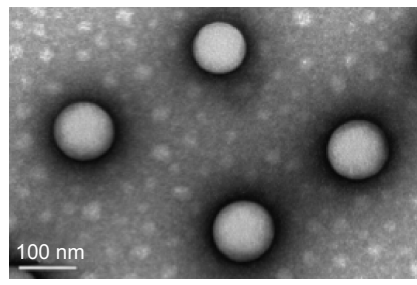

B

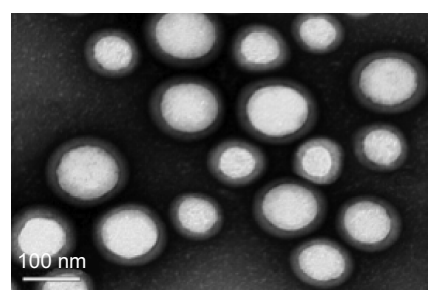

C

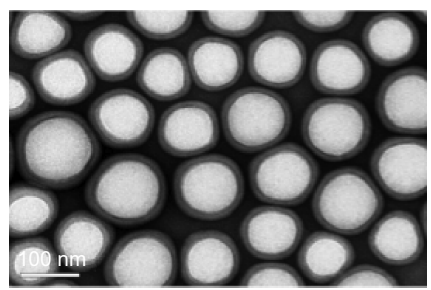

D

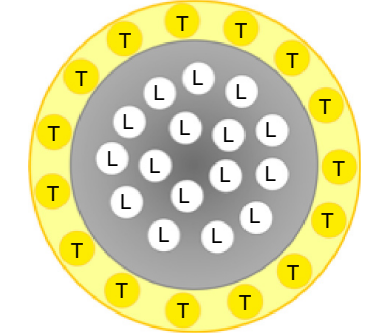

PLGA

105.5

\section{PLGA-chitosan}

107.8

\section{PLGA-lovastatin- chitosan-tetracycline} 111.5

T: Tetracycline

L: Lovastatin

- PLGA

: Chitosan

\section{Schematic diagram of PLGA-lovastatin-chitosan- tetracycline}

Figure I TEM photographs of (A) PLGA, (B) PLGA-chitosan, and (C) PLGA-lovastatin-chitosan-tetracycline nanoparticles. (D) The schematic diagram of PLGA-lovastatinchitosan-tetracycline nanoparticles.

Abbreviations: PLGA, poly(D,L-lactide-co-glycolide acid); TEM, transmission electron microscopy.

PLGA-lovastatin-chitosan-tetracycline $0.3 \%$ (Figure 6B), compared with PLGA-lovastatin-chitosan and PLGAchitosan on days $0,1,3,5$, and 7 . No inhibition zone was observed in the PLGA-lovastatin-chitosan-tetracycline $0.1 \%$, PLGA-lovastatin-chitosan, and PLGA-chitosan groups. However, an inhibition zone was clearly observed for A. actinomycetemcomitans and $P$. nigrescens on days 1,3 , 5 , and 7 in the PLGA-lovastatin-chitosan-tetracycline $0.3 \%$ group. Figure $6 \mathrm{C}$ shows the diameter of the inhibition zone of $A$. actinomycetemcomitans and $P$. nigrescens treated with PLGA-lovastatin-chitosan-tetracycline $0.3 \%$ in relation to the number of days of incubation. PLGA-lovastatin-chitosantetracycline $0.3 \%$ had an inhibitory effect at the first day of incubation, which continued to day 7 .

\section{Regeneration of periodontal tissue and new bone formation in dogs}

Tissue blocks containing the mesial artificial defect of the maxillary canine were harvested from both experimental groups (groups where the defect was filled with nanoparticles) and the control group (where the defect was left unfilled) at 8 weeks after surgery. Sagittal sections along the mesiodistal direction were prepared and routine hematoxylin and eosin staining was performed for histopathological examination. No evident inflammation or foreign body reaction was observed in either experimental group (Figure 7A and C) or control group (Figure 7B and D), indicating biocompatibility in vivo. New deposits of cementum on the root surface and cementoid materials in the surgical defect were present in 
A

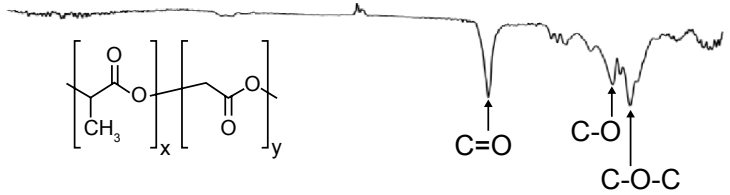

B

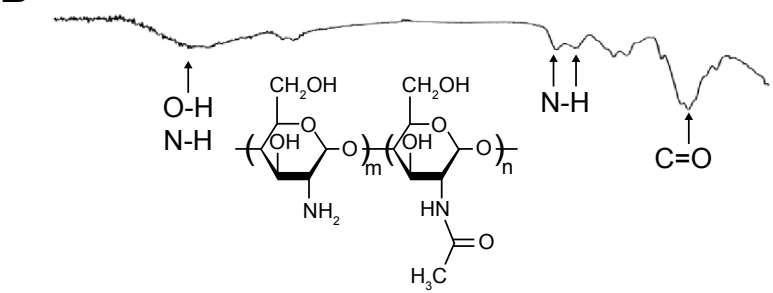

C

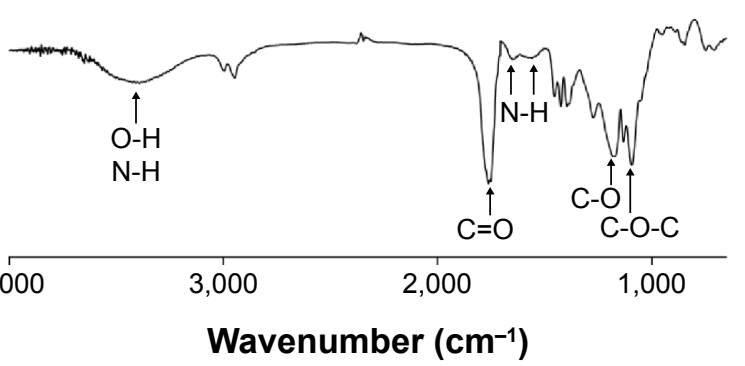

Figure 2 IR spectra of (A) PLGA, (B) chitosan, and (C) PLGA-chitosan nanoparticles.

Note: Characteristic peaks of PLGA-chitosan were observed by IR spectra. Abbreviations: IR, infrared; PLGA, poly(D,L-lactide-co-glycolide acid). the experimental group, in contrast to pure dense fibrous tissue in the control group. Active plasmacytoid osteoblastic rimming along the trabecular surface of the bone adjacent to the defect was prominent in the experimental group (high magnification in Figure 7E). However, osteoblasts lining the bone surface in the control group had a flattened morphology, implying a relatively low level of osteogenic activity (Figure 7F). Figure 8 shows a statistically significant difference $(P=0.04)$ in the percentage of new bone formation between the experimental (41.32\%) and control (34.01\%) groups by micro-CT analysis.

\section{Discussion}

Mechanical debridement of periodontal pockets by scaling and root planing is the standard treatment for chronic periodontitis. ${ }^{34}$ However, mechanical debridement is limited for eliminating pathogens in furcations and deep pockets because these sites are difficult to access. ${ }^{35}$ The local subgingival application of tetracycline as an adjunct to mechanical debridement was developed and pocket depth reduction by local antimicrobial application was demonstrated in deep or recurrent periodontal sites. ${ }^{36}$ Chronic periodontitis is not only an infection process but

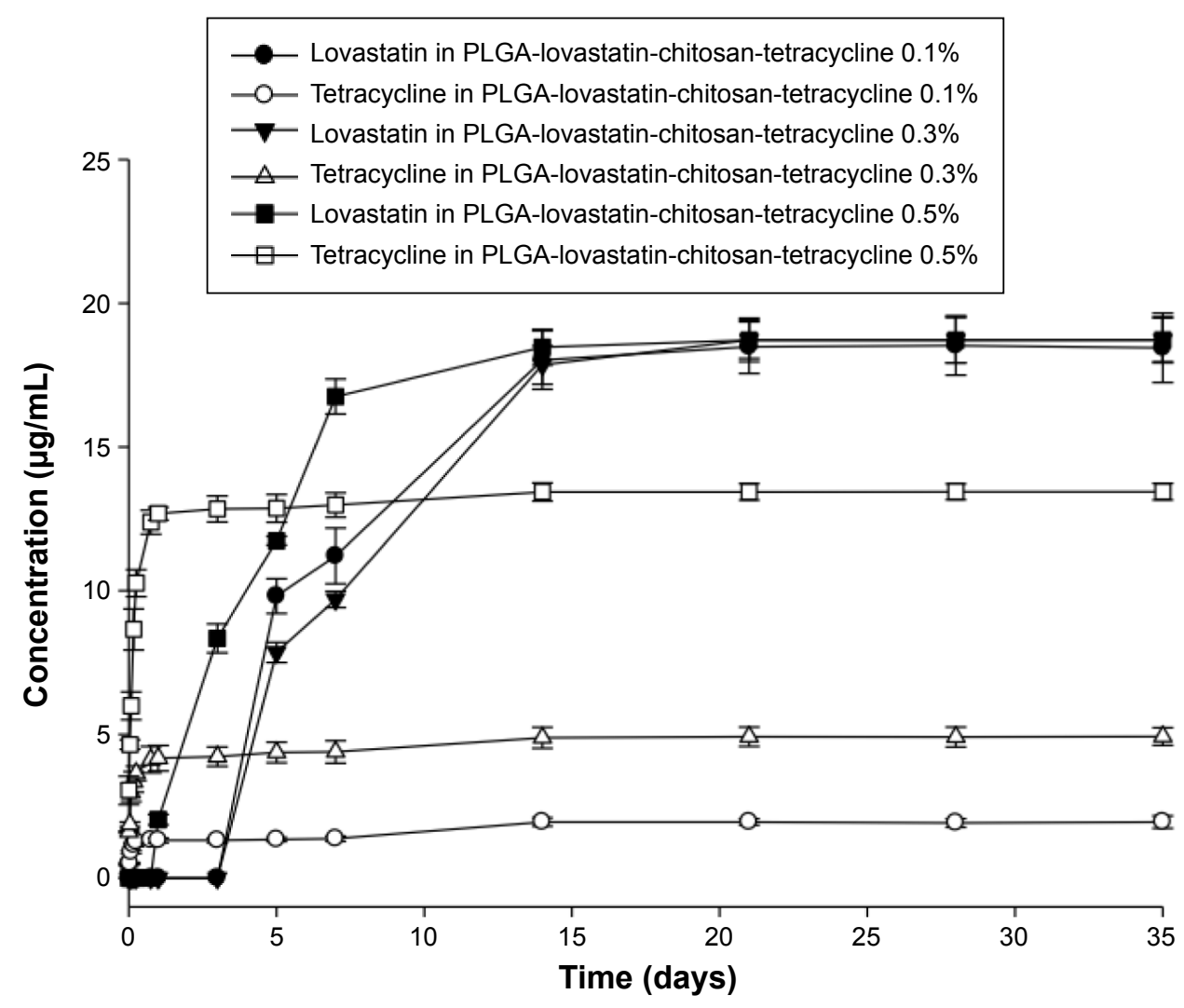

Figure 3 Burst release of tetracycline was observed on day I followed by a gradual release until day I4.

Note: Lovastatin release was slower than for tetracycline and the prominent release of lovastatin was detected until day $2 \mathrm{I}$.

Abbreviation: PLGA, poly(D,L-lactide-co-glycolide acid). 


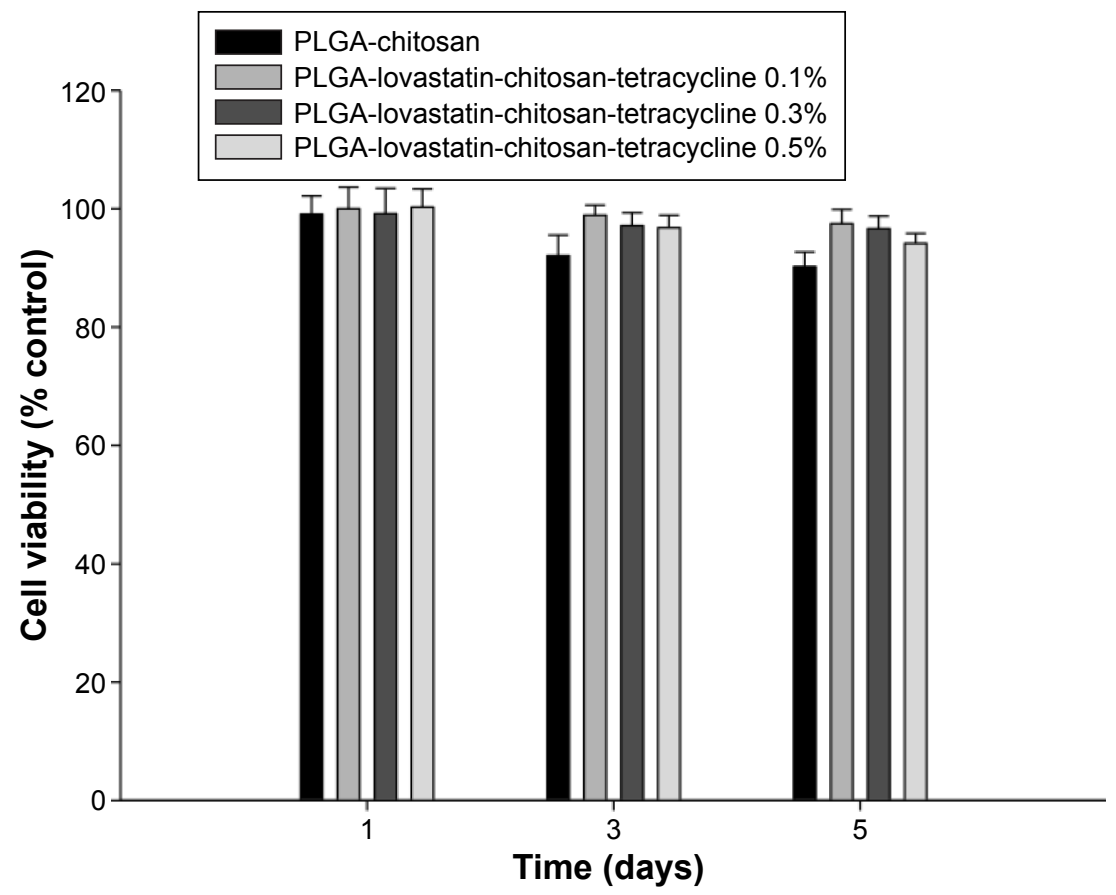

Figure 4 Cell viability of PLGA-chitosan and PLGA-lovastatin-chitosan-tetracycline $0.1 \%, 0.3 \%$, and $0.5 \%$ at I, 3 , and 5 days of culture by MTT assay Abbreviations: MTT, 3-(4,5-dimethylthiazol-2-yl)-2,5-diphenyltetrazolium bromide; PLGA, poly(D,L-lactide-co-glycolide acid).

also involves inflammation and tissue loss. ${ }^{37}$ Consequently, the use of tetracycline to control infection is not sufficient; therefore, the combination of tetracycline with lovastatin to enhance bone regeneration is anticipated. A previous study using PLGA nanoparticles as a carrier to control lovastatin release showed that this method enhanced bone repair in rats. ${ }^{28}$ In this study, a double emulsion was utilized to add a chitosan coating on PLGA nanoparticles. The particle size (111.5 nm) of PLGA-lovastatin-chitosan-tetracycline nanoparticles was slightly greater than that $(105.5 \mathrm{~nm})$ of PLGA

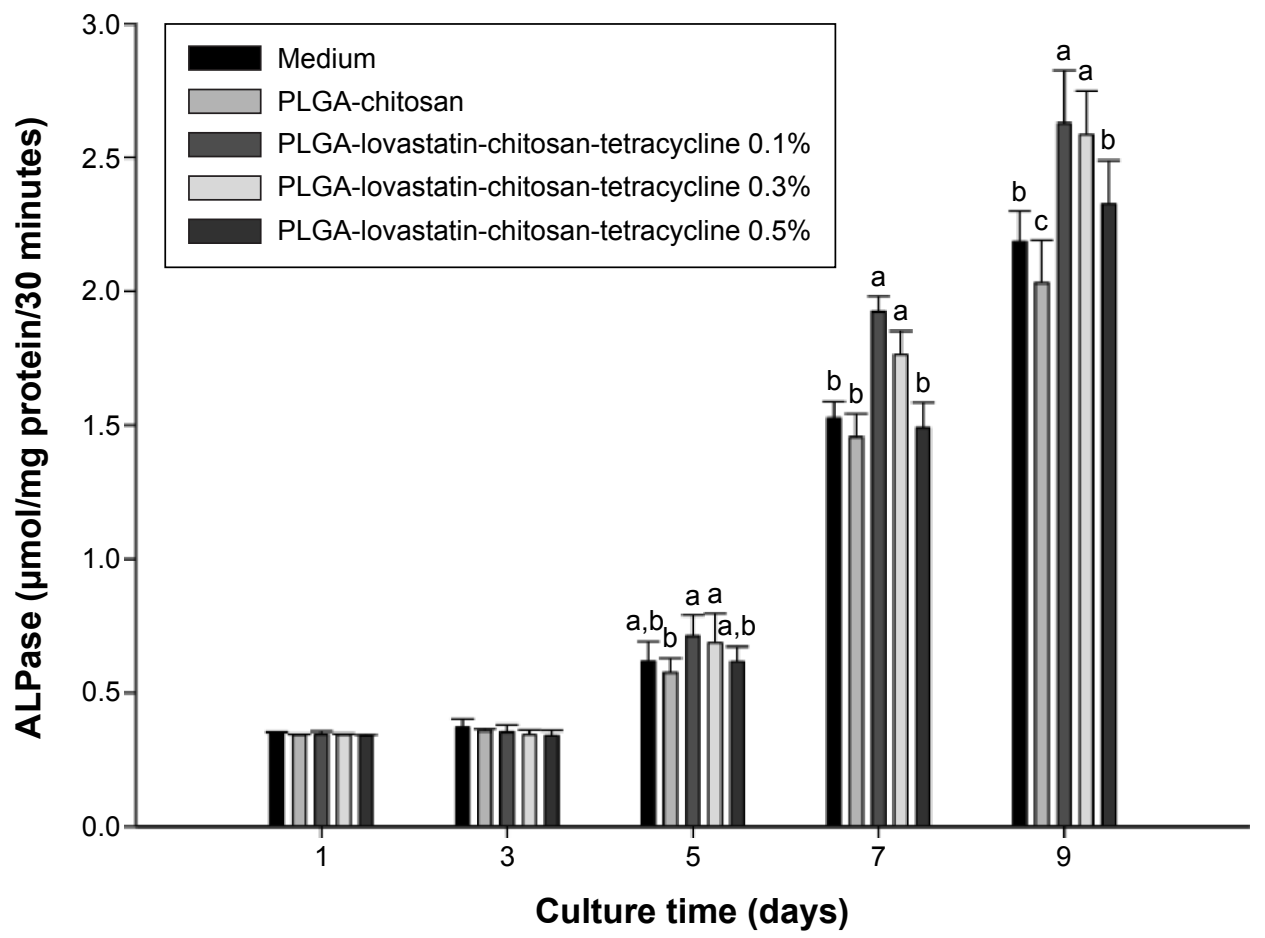

Figure 5 Osteogenic potential of PLGA-chitosan and PLGA-lovastatin-chitosan-tetracycline $0.1 \%, 0.3 \%$, and $0.5 \%$ on days I, 3, 5, 7, and 9 of culture. Note: Significant differences were represented with different letters $(a, b, c)$.

Abbreviations: ALPase, alkaline phosphatase; PLGA, poly(D,L-lactide-co-glycolide acid). 
A

A. a

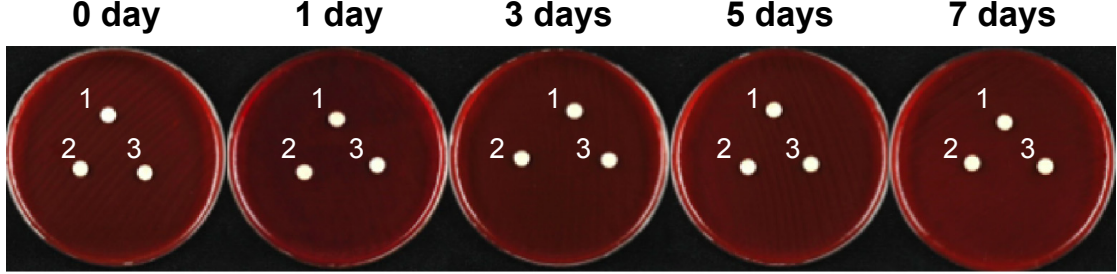

P. $\mathbf{n}$

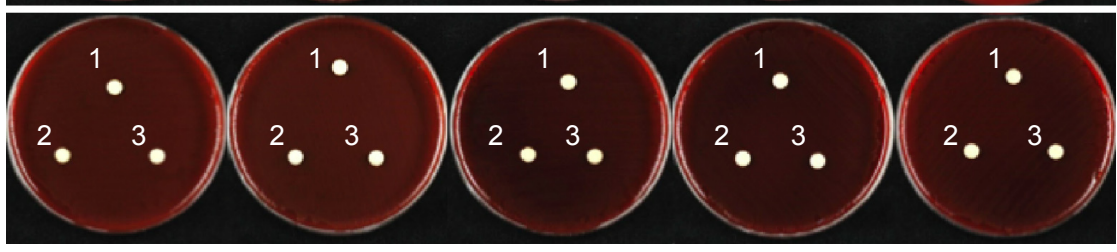

B

A. a

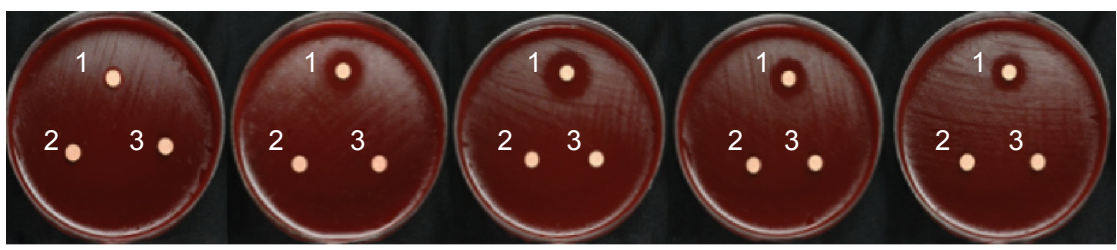

P. $\mathbf{n}$
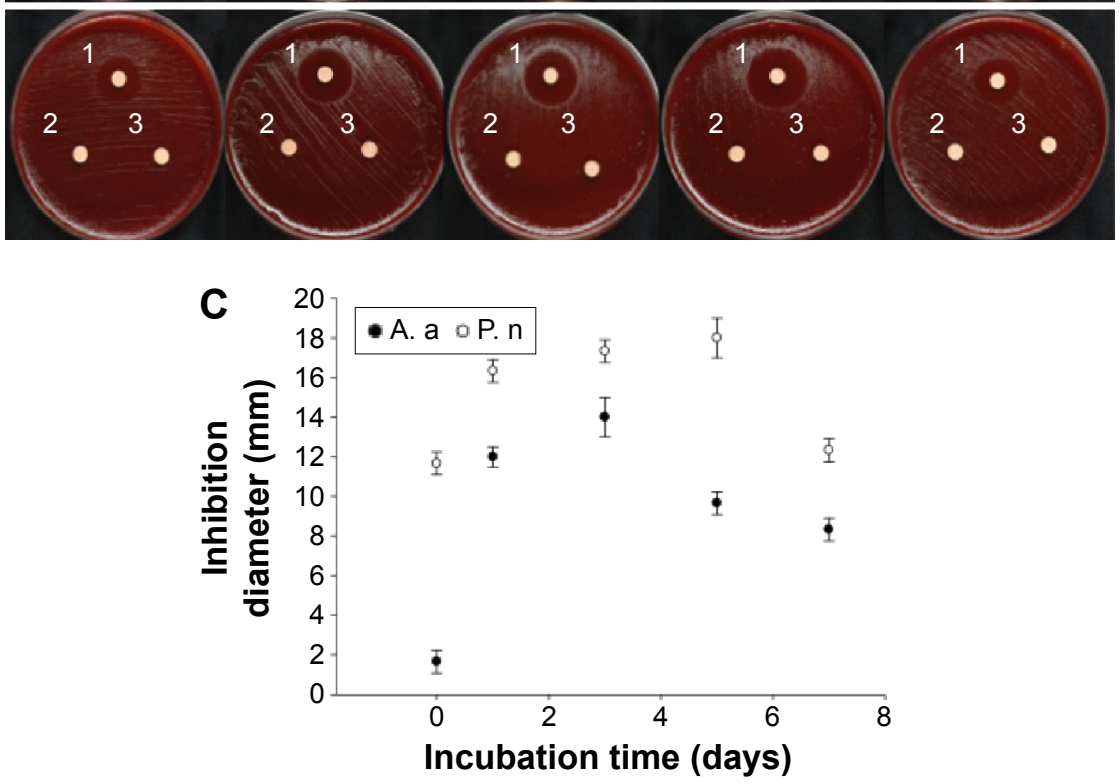

Figure 6 Inhibition zone of Aggregatibacter actinomycetemcomitans (A. a) and Prevotella nigrescens (P. n) growth using (A) PLGA-lovastatin-chitosan-tetracycline 0.1\% (I: PLGA-lovastatin-chitosan-tetracycline 0.1\%, compared with 2: PLGA-lovastatin-chitosan, and 3: PLGA-chitosan) and (B) PLGA-lovastatin-chitosan-tetracycline 0.3\% (I: PLGA-lovastatin-chitosan-tetracycline $0.3 \%$, compared with 2: PLGA-lovastatin-chitosan, and 3: PLGA-chitosan) at 0, I, 3, 5, or 7 days. (C) Diameters of the inhibition zone of $A$. actinomycetemcomitans and $P$. nigrescens using PLGA-lovastatin-chitosan-tetracycline $0.3 \%$ in relation to incubation days.

Abbreviation: PLGA, poly(D,L-lactide-co-glycolide acid).

nanoparticles (Figure 1). To confirm the halo surrounding PLGA nanoparticles was composed of chitosan, Fourier transform infrared spectroscopy was used to examine the functional groups of PLGA-chitosan nanoparticles. The spectra of PLGA-chitosan contained characteristic peaks of chitosan $\left(3,336,1,649\right.$, and 1,560 $\left.\mathrm{cm}^{-1}\right)$ (Figure 2) demonstrating the outer structure was chitosan.

Infection during chronic periodontitis must be controlled before bone regeneration can take place. Therefore, tetracycline was designed to be released at the beginning of treatment, followed by lovastatin. The release profile of tetracycline showed that a large amount was released on the first day of administration followed by a gradual release until day 14 (Figure 3). The amount released increased when higher concentrations were used, and a higher concentration was more effective against A. actinomycetemcomitans and $P$. nigrescens. However, the $0.5 \%$ tetracycline group exhibited a significantly lower amount of ALP on days 7 and 9 

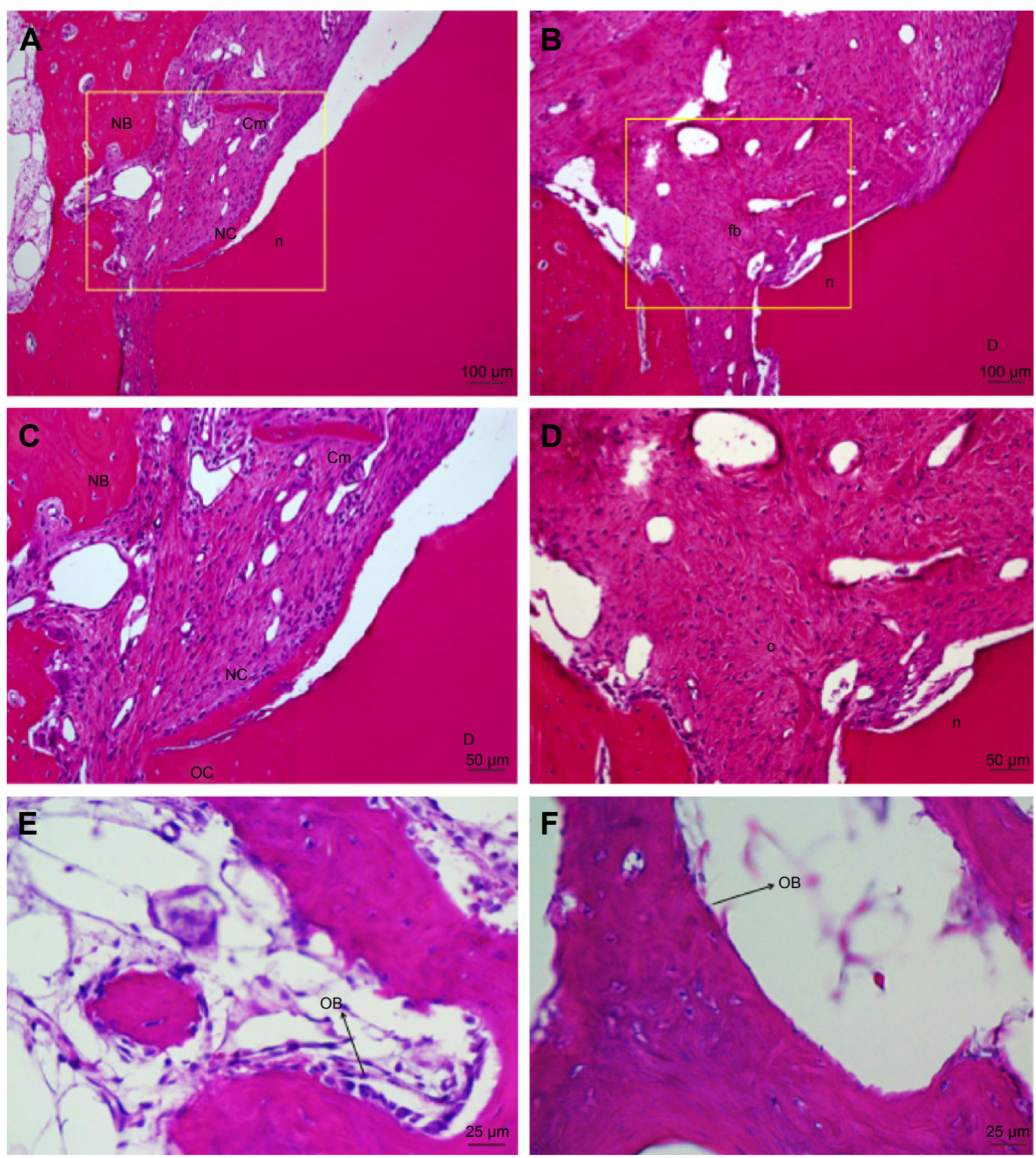

Figure 7 Histologic photomicrographs of the mesiodistal section of defects in experimental (A, C, E) and control (B, D, F) sites in beagle dogs.

Notes: (A) In the experimental site, residual biomaterials were not observed, and there were no signs of inflammatory cell infiltration. New cementum and connective tissue were observed at the notch area. (C) Higher magnification of the framed area in (A). Newly formed cementum (NC) was observed on the denuded root surface, and free cementoid-like tissue $(\mathrm{Cm})$ was found inside the connective tissue. The coronal end of the old cementum (OC) is shown. (B) Fibrous tissue (fb) occupying the notch area in the control group. Mild root resorption was observed at the coronal portion of the notch. (D) Higher magnification of the framed area in (B). (E) Osteoblasts (OB) in the experimental site showed various shapes from cuboidal to tall columnar with basally located nuclei and had high osteogenic activity. (F) In the control site, the OB were flat with nonprominent nuclei.

Abbreviations: NB, new bone; D, dentin; n, notch; c, connective tissue.

compared with the other groups (Figure 5), even though the cell viability was not different among the three groups (Figure 4). In addition, a higher tetracycline dose was demonstrated to reduce cellular differentiation and BMP-2 expression. ${ }^{38}$ Therefore, the $0.3 \%$ tetracycline group was selected for the animal experiment in the current study.

Lovastatin was released from day 3 in the $0.1 \%$ and $0.3 \%$ tetracycline groups and a burst release was observed until day 14 , followed by a gradual release until day 21 (Figure 3). Although numerous studies have demonstrated the controlled release of lovastatin, the optimal release profile has not been determined. A previous study using PLGA nanoparticles as a carrier showed $97 \%$ release after 7 days and this release pattern was effective for inducing bone regeneration. ${ }^{28}$ In the current study, the release duration of lovastatin was prolonged and had a significant effect in enhancing periodontal bone regeneration (Figure 8). The mechanisms of drug release from polymer encapsulation include diffusion and polymer degradation. ${ }^{39}$ As the tetracycline was incorporated at the outer chitosan layer and most of the encapsulated 


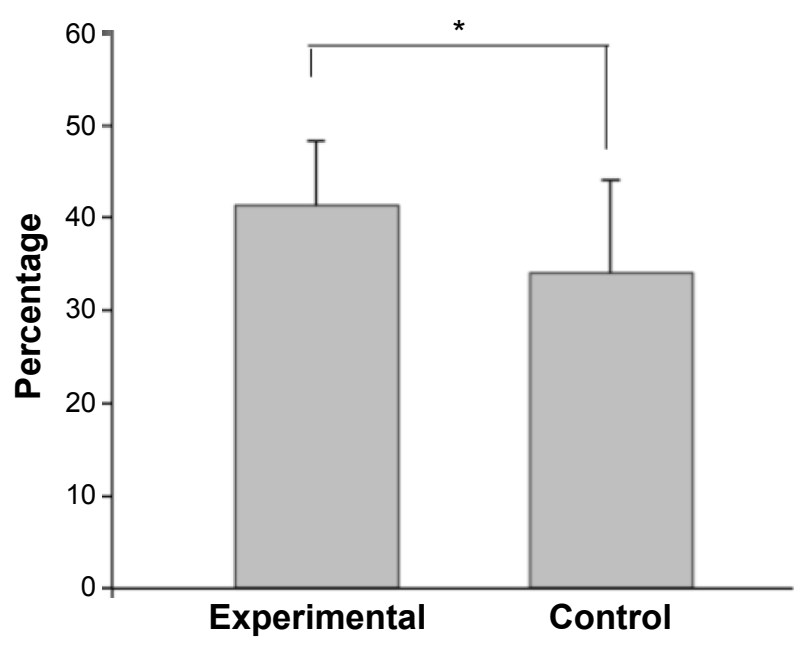

Figure 8 Percentage of new bone formation by micro-CT analysis. Note: The new bone formation was significantly higher in the experimental group than that in the control group. Significant difference was represented with *.

tetracycline was released on the first day, its release was basically from diffusion. In contrast, lovastatin was incorporated in the inner PLGA layer, and its release after tetracycline might be from diffusion and polymer degradation. The PLGA molecular chains would exhibit decreased molecular weight due to hydrolysis, causing the increase in the free volume in PLGA nanoparticles. When the free volume is larger than the size of lovastatin, the lovastatin can penetrate through the PLGA shell due to the concentration gradient. PLGA-lovastatin-chitosan-tetracycline nanoparticles can be adjusted to control the release profile of tetracycline and lovastatin for their future application to treat periodontitis of different severity. For example, lovastatin release can be decelerated by selecting higher lactic units, higher crystallinity, and a higher mean molecular weight of PLGA. ${ }^{40}$ The degradation rate of chitosan is inversely proportional to the degree of deacetylation. ${ }^{41}$ Consequently, using a higher degree of chitosan deacetylation could prolong tetracycline release. Furthermore, a hydrophilic material can be used to coat the outer surfaces of chitosan to decelerate the release of lovastatin and tetracycline if a longer duration of drug release is necessary.

The progression of periodontitis and its recovery are a complex process. Lovastatin and tetracycline were selected as drugs for local release adjunctive treatments because infection control and bone regeneration are considered the most decisive steps of treatment outcomes. Sundararaj et $\mathrm{al}^{42}$ designed a more complex multiple drug delivery system that included metronidazole, ketoprofen, doxycycline, and simvastatin that exhibited antibacterial, anti-inflammatory, antiresorptive, and osteogenic functions. However, they did not conduct an in vitro evaluation or animal study, thus we cannot compare our preparations with theirs.

To investigate the regenerative potential of PLGA-lovastatin-chitosan-tetracycline $0.3 \%$ nanoparticles, we used a three-walled defect canine model because of its flowable and injectable properties. ${ }^{43}$ In vitro results show that PLGA-chitosan nanoparticles have neither osteogenic potential (Figure 5) nor bactericidal activity (Figure 6), and this is the reason that we left defects unfilled instead of placing PLGA-chitosan nanoparticles in the control group. Histologic analysis revealed no signs of acute inflammation or foreign body reactions, and superior bone and cementum formation was observed in the experimental group. In addition, high osteogenic activity was also observed in the experimental group indicating the regenerative potential of this material. Although histomorphometry provides high resolution and direct representations of alveolar bone levels, there are obvious limitations, such as tissue sample destruction and challenges in three-dimensional image reconstruction. ${ }^{44}$ In contrast, micro-CT provides significant advantage for identifying mineralized structures with high accuracy and reproducibility. In this study, we used micro-CT to quantify new bone formation and volumetric analysis. The results were consistent with histologic analysis, indicating the superior bone regenerative potential of PLGA-lovastatinchitosan-tetracycline nanoparticles.

Local drug delivery systems are more favorable compared to a systemic approach because of multiple advantages, including site-specific delivery, reduction in gastrointestinal side effects, decreased dosage frequency, and minimized chance of bacterial resistance. ${ }^{45}$ They can be used as an effective adjunctive treatment in addition to scaling and root planing. Pradeep et al ${ }^{29,30}$ designed a biodegradable controlled-release gel containing simvastatin and observed significant probing depth reduction, clinical attachment level gain, and radiographic bone fill in chronic periodontitis patients. The same drug delivery device was applied for treating type II diabetes patients with chronic periodontitis and significantly improved the clinical outcomes of scaling and root planing. ${ }^{31}$ In this study, PLGA-lovastatin-chitosan-tetracycline nanoparticles showed good biocompatibility and osteogenic potential, and significantly increased new bone formation in three-walled defects in a beagle model. Human randomized case-controlled clinical trial will be warranted in future studies.

\section{Conclusion}

PLGA-lovastatin-chitosan-tetracycline nanoparticles were successfully prepared using a double emulsion method and showed an effective controlled-release pattern, good 
biocompatibility, superior osteogenic potential, and antibacterial activity. They also promoted new bone formation in three-walled defects in beagle dogs. Therefore, this local delivery device could be used as a novel adjunctive treatment in periodontitis.

\section{Acknowledgments}

This study was supported by grants (103-2314-B-002-093MY3 and 103-2314-B-002-109) from the Ministry of Science and Technology and grants (103-S2395 and 102-N2260) from National Taiwan University Hospital, Taiwan.

\section{Disclosure}

The authors report no conflicts of interest in this work.

\section{References}

1. Pihlstrom BL, Michalowicz BS, Johnson NW. Periodontal diseases. Lancet. 2005;366(9499):1809-1820.

2. Petersen PE, Ogawa H. Strengthening the prevention of periodontal disease: the WHO approach. J Periodontol. 2005;76(12):2187-2193.

3. Aljateeli M, Koticha T, Bashutski J, et al. Surgical periodontal therapy with and without initial scaling and root planing in the management of chronic periodontitis: a randomized clinical trial. J Clin Periodontol. 2014;41(7):693-700.

4. Caton J, Nyman S, Zander H. Histometric evaluation of periodontal surgery. II. Connective tissue attachment levels after four regenerative procedures. J Clin Periodontol. 1980;7(3):224-231.

5. Susin C, WikesjöUM. Regenerative periodontal therapy: 30 years of lessons learned and unlearned. Periodontol 2000. 2013;62(1):232-242.

6. Slots J, Mashimo P, Levine MJ, Genco RJ. Periodontal therapy in humans. I. Microbiological and clinical effects of a single course of periodontal scaling and root planing, and of adjunctive tetracycline therapy. J Periodontol. 1979;50(10):495-509.

7. Slots J, Rams TE. Antibiotics in periodontal therapy: advantages and disadvantages. J Clin Periodontol. 1990;17(s1):479-493.

8. Slots J, Ting M. Systemic antibiotics in the treatment of periodontal disease. Periodontol 2000. 2002;28:106-176.

9. Goodson JM, Offenbacher S, Farr DH, Hogan PE. Periodontal disease treatment by local drug delivery. J Periodontol. 1985;56(5): $265-272$.

10. Greenstein G, Polson A. The role of local drug delivery in the management of periodontal diseases: a comprehensive review. J Periodontol. 1998; 69(5):507-520.

11. Rams TE, Slots J. Local delivery of antimicrobial agents in the periodontal pocket. Periodontol 2000. 1996;10:139-159.

12. Giri TK, Thakur A, Alexander A, Ajazuddin, Badwaik H, Tripathi DK. Modified chitosan hydrogels as drug delivery and tissue engineering systems: present status and applications. Acta Pharmacol Sin B. 2012; 2(5):439-449.

13. Wang JJ, Zeng ZW, Xiao RZ, et al. Recent advances of chitosan nanoparticles as drug carriers. Int J Nanomedicine. 2011;6:765-774.

14. Arancibia R, Maturana C, Silva D, et al. Effects of chitosan particles in periodontal pathogens and gingival fibroblasts. J Dent Res. 2013; 92(8):740-745.

15. Ozmeric N, Ozcan G, Haytac CM, Alaaddinoglu EE, Sargon MF, Senel S. Chitosan film enriched with an antioxidant agent, taurine, in fenestration defects. J Biomed Mater Res. 2000;51(3):500-503.

16. Pichayakorn W, Boonme P. Evaluation of cross-linked chitosan microparticles containing metronidazole for periodontitis treatment. Mater Sci Eng C Mater Biol Appl. 2013;33(3):1197-1202.
17. Akncbay H, Senel S, Ay ZY. Application of chitosan gel in the treatment of chronic periodontitis. J Biomed Mater Res B Appl Biomater. 2007; 80(2):290-296.

18. Hebert PR, Gaziano JM, Chan KS, Hennekens CH. Cholesterol lowering with statin drugs, risk of stroke, and total mortality: an overview of randomized trials. JAMA. 1997;278(4):313-321.

19. Nissen SE, Tuzcu EM, Schoenhagen P, et al. Statin therapy, LDL cholesterol, C-reactive protein, and coronary artery disease. $N$ Engl J Med. 2005;352(1):29-38.

20. Kataria P, Kaur J, Parvez E, Maurya R. Statins: the paradigm shift in periodontal regeneration. SRM J Res Dent Sci. 2014;5(1):26.

21. Mundy G, Garrett R, Harris S, et al. Stimulation of bone formation in vitro and in rodents by statins. Science. 1999;286(5446):1946-1949.

22. Cunha-Cruz J, Saver B, Maupome G, Hujoel PP. Statin use and tooth loss in chronic periodontitis patients. J Periodontol. 2006;77(6): 1061-1066.

23. Seto H, Ohba H, Tokunaga K, Hama H, Horibe M, Nagata T. Topical administration of simvastatin recovers alveolar bone loss in rats. $J$ Periodontal Res. 2008;43(3):261-267.

24. Makadia HK, Siegel SJ. Poly lactic-co-glycolic acid (PLGA) as biodegradable controlled drug delivery carrier. Polymers. 2011;3(3): 1377-1397.

25. Kashi TSJ, Eskandarion S, Esfandyari-Manesh M, et al. Improved drug loading and antibacterial activity of minocycline-loaded PLGA nanoparticles prepared by solid/oil/water ion pairing method. Int J Nanomedicine. 2012;7:221-234.

26. Nath SD, Linh NT, Sadiasa A, Lee BT. Encapsulation of simvastatin in PLGA microspheres loaded into hydrogel loaded BCP porous spongy scaffold as a controlled drug delivery system for bone tissue regeneration. J Biomater Appl. 2014;28(8):1151-1163.

27. Naito Y, Terukina T, Galli S, et al. The effect of simvastatin-loaded polymeric microspheres in a critical size bone defect in the rabbit calvaria. Int J Pharm. 2014;461(1):157-162.

28. Ho MH, Chiang CP, Liu YF, et al. Highly efficient release of lovastatin from poly(lactic-co-glycolic acid) nanoparticles enhances bone repair in rats. J Orthop Res. 2011;29(10):1504-1510.

29. Pradeep AR, Priyanka N, Kalra N, Naik SB, Singh SP, Martande S. Clinical efficacy of subgingivally delivered $1.2-\mathrm{mg}$ simvastatin in the treatment of individuals with Class II furcation defects: a randomized controlled clinical trial. J Periodontol. 2012;83(12):1472-1479.

30. Pradeep AR, Thorat MS. Clinical effect of subgingivally delivered simvastatin in the treatment of patients with chronic periodontitis: a randomized clinical trial. J Periodontol. 2010;81(2):214-222.

31. Pradeep AR, Rao NS, Bajaj P, Kumari M. Efficacy of subgingivally delivered simvastatin in the treatment of patients with type 2 diabetes and chronic periodontitis: a randomized double-masked controlled clinical trial. J Periodontol. 2013;84(1):24-32.

32. Terriza A, Vilches-Perez JI, de la Orden E, et al. Osteoconductive potential of barrier nanoSiO ${ }_{2}$ PLGA membranes functionalized by plasma enhanced chemical vapour deposition. Biomed Res Int. 2014; 2014:253590.

33. Mukhopadhyay P, Sarkar K, Bhattacharya S, Bhattacharyya A, Mishra R, Kundu PP. pH sensitive N-succinyl chitosan grafted polyacrylamide hydrogel for oral insulin delivery. Carbohydr Polym. 2014;112:627-637.

34. Hung HC, Douglass CW. Meta-analysis of the effect of scaling and root planing, surgical treatment and antibiotic therapies on periodontal probing depth and attachment loss. J Clin Periodontol. 2002;29(11): 975-986.

35. Greenstein G. Nonsurgical periodontal therapy in 2000: a literature review. J Am Dent Assoc. 2000;131(11):1580-1592.

36. Matesanz-Perez P, Garcia-Gargallo M, Figuero E, Bascones-Martinez A, Sanz M, Herrera D. A systematic review on the effects of local antimicrobials as adjuncts to subgingival debridement, compared with subgingival debridement alone, in the treatment of chronic periodontitis. J Clin Periodontol. 2013;40(3):227-241. 
37. Thomas MV, Puleo DA. Infection, inflammation, and bone regeneration: a paradoxical relationship. J Dent Res. 2011;90(9):1052-1061.

38. Park JB. Effects of doxycycline, minocycline, and tetracycline on cell proliferation, differentiation, and protein expression in osteoprecursor cells. J Craniofac Surg. 2011;22(5):1839-1842.

39. Gopferich A. Mechanisms of polymer degradation and erosion. Biomaterials. 1996;17(2):103-114.

40. Alexis F. Factors affecting the degradation and drug-release mechanism of poly(lactic acid) and poly[(lactic acid)-co-(glycolic acid)]. Polym Int 2005;54(1):36-46.

41. Tomihata $\mathrm{K}$, Ikada $\mathrm{Y}$. In vitro and in vivo degradation of films of chitin and its deacetylated derivatives. Biomaterials. 1997;18(7):567-575.
42. Sundararaj SC, Thomas MV, Peyyala R, Dziubla TD, Puleo DA Design of a multiple drug delivery system directed at periodontitis. Biomaterials. 2013;34(34):8835-8842.

43. Hayashi C, Kinoshita A, Oda S, Mizutani K, Shirakata Y, Ishikawa I. Injectable calcium phosphate bone cement provides favorable space and a scaffold for periodontal regeneration in dogs. $J$ Periodontol. 2006; 77(6):940-946.

44. Park CH, Abramson ZR, Taba M Jr, et al. Three-dimensional microcomputed tomographic imaging of alveolar bone in experimental bone loss or repair. J Periodontol. 2007;78(2):273-281.

45. Joshi D, Garg T, Goyal AK, Rath G. Advanced drug delivery approaches against periodontitis. Drug Deliv. 2014:1-15.
International Journal of Nanomedicine

\section{Publish your work in this journal}

The International Journal of Nanomedicine is an international, peerreviewed journal focusing on the application of nanotechnology in diagnostics, therapeutics, and drug delivery systems throughout the biomedical field. This journal is indexed on PubMed Central, MedLine, CAS, SciSearch ${ }^{\circledR}$, Current Contents ${ }^{\circledR} /$ Clinical Medicine,

\section{Dovepress}

Journal Citation Reports/Science Edition, EMBase, Scopus and the Elsevier Bibliographic databases. The manuscript management system is completely online and includes a very quick and fair peer-review system, which is all easy to use. Visit http://www.dovepress.com/ testimonials.php to read real quotes from published authors.

Submit your manuscript here: http://www.dovepress.com/international-journal-of-nanomedicine-journal 